\title{
Tumor adenomatoide de túnica albugínea. Caso clínico
}

\section{Adenomatoid tumor of the tunica albuginea. A case report}

\section{Sr. Director:}

Los tumores paratesticulares representan un escaso porcentaje de los tumores intraescrotales. Histológicamente se caracterizan por ser tumores benignos derivados del mesénquima. De ellos, la forma más frecuente es el tumor adenomatoide, seguido del cistadenoma papilar y el leiomioma. Estos tumores pueden presentarse en muchos órganos y en ambos sexos, aunque son más frecuentes en el varón y en el área genital. El tratamiento de elección es la extirpación local de la lesión. Si existen dudas sobre la malignidad se realiza biopsia extemporánea y orquiectomía en caso de confirmarse esta condición.

Describimos un caso clínico de tumoración paratesticular. El diagnóstico de estas neoformaciones es poco frecuente y se presenta habitualmente entre la tercera y quinta décadas de la vida ${ }^{1}$. La mayoría de estos tumores son de naturaleza benigna $(80 \%)^{1}$. En general, se localizan en el epidídimo, aunque hay casos más raros localizados en la túnica albugínea, en los cordones espermáticos o incluso en la próstata ${ }^{2}$.

El caso que presentamos es de un varón de 28 años, sin antecedentes médico-quirúrgicos de interés, que acudió a Urgencias por tumoración escrotal izquierda, dolorosa, de una semana de evolución.

En la exploración física el pene y los testes eran normales. En la cola del epidídimo izquierdo se detectó un nódulo de consistencia aumentada y doloroso a la palpación.

La ecografía escrotal mostró el parénquima del testículo izquierdo normal y una imagen redondeada, de $1 \mathrm{~cm}$, que parecía depender de las cubiertas testiculares o del epidídimo. El paciente fue tratado con antiinflamatorios y derivado a consultas externas de Urología para completar su estudio con resonancia magnética (RM).
En la RM destacaba hacia la porción caudal y lateral del testículo izquierdo una lesión extratesticular de $7 \mathrm{~mm}$ de diámetro isointensa, con el parénquima testicular en T1, hipointensa en $\mathrm{T} 2$ y con intensa captación de contraste, que parece tener relación con la zona de la cola del epidídimo y que se sitúa en la zona más lateral del teste ${ }^{3}$, siendo el diagnóstico más probable el de un tumor fibroso adenomatoide.

Los marcadores tumorales resultaron normales.

Ante estos hallazgos se realizó punción-aspiración con aguja fina (PAAF) de la lesión, donde se observó escasa celularidad de aspecto epitelial-mesotelial, con poca o nula atipia nuclear que citológicamente sugiere un tumor adenomatoide.

Se realizó la exéresis de la neoformación, que se localizaba en la túnica albugínea, previa biopsia extemporánea de la misma que fue informada de neoformación adenomatoide con ausencia de malignidad. El resultado anatomopatológico fue de tumor adenomatoide de túnica albugínea.

A los dos años de seguimiento el paciente se encuentra libre de enfermedad.

Estos tumores representan hasta el 5\% de las masas intraescrotales ${ }^{4}$, y es la forma de presentación más frecuente (60\%) de los tumores paratesticulares. No es un tumor estrictamente urológico, ya que se puede presentar en otros órganos como el útero o las trompas de Falopio. No obstante, está descrita una tendencia a su localización en órganos pélvicos, así como una mayor frecuencia de aparición en el varón, aunque puede presentarse en cualquier órgano de la economía 5 .

La localización habitual es el epidídimo, principalmente en la cola. Nuestro paciente presentaba la lesión en la túnica albugínea, donde está descrita una incidencia del 14\%. Otras 


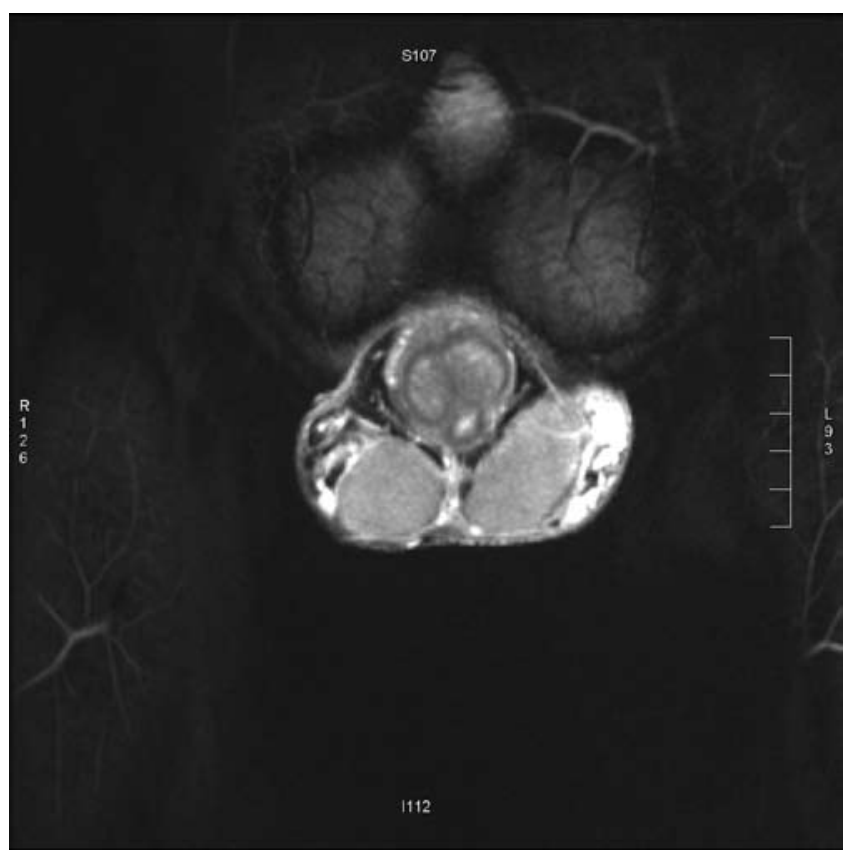

Figura 1 - Ecografía en la que se identifica la lesión paratesticular.

localizaciones menos usuales son los cordones espermáticos o la próstata 4 .

La forma de presentación habitual es la de masa indolora, detectada de forma casual por el propio paciente ${ }^{1}$. Menos frecuente es la presentación como masa dolorosa, como sería el caso que nos ocupa. Clínicamente, el tamaño en el momento del diagnóstico suele oscilar entre los 2 y $3 \mathrm{~cm}^{6}$. En nuestro caso el tamaño fue algo menor, de $1 \mathrm{~cm}$, probablemente porque se trató de una tumoración sintomática.

Entre las pruebas de imagen la ecografía escrotal es útil para identificar el origen de la lesión, pues diferencia claramente las lesiones testiculares de las paratesticulares ${ }^{7}$. No obstante, esta técnica pierde eficacia a la hora de filiar el origen exacto de la lesión. En nuestro caso la ecografía sirvió para identificar la lesión como paratesticular, aunque no para discernir si el origen era el epidídimo o las cubiertas testiculares (fig. 1). Para ello disponemos de tomografía axial computarizada y RM, que delimitan mucho mejor los tejidos blandos y las estructuras escrotales ${ }^{8}$.

Uno de los temas más debatidos en este ámbito es el uso o no de PAAF. Autores como Flores et $\mathrm{al}^{9}$ prefieren no realizarla, debido al riesgo de diseminación en caso de malignidad de la lesión. No obstante, dado que hasta el $80 \%$ de las masas paratesticulares son benignas, otros autores indican esta técnica para realizar un diagnóstico diferencial con otras entidades y completarlo ${ }^{10}$. Nosotros, dados los resultados de la RM optamos por realizarla, observando datos que orientaban hacia el diagnóstico de tumor adenomatoide (fig. 2).

En cuanto al tratamiento hay consenso en que al ser lesiones benignas es suficiente con la exéresis local de la lesión. En nuestro caso realizamos una biopsia extemporánea del tumor para confirmar la ausencia de malignidad, dado que de no haber sido este el diagnóstico la actitud terapéutica habría cambiado, teniendo que realizar en ese caso una orquiectomía.

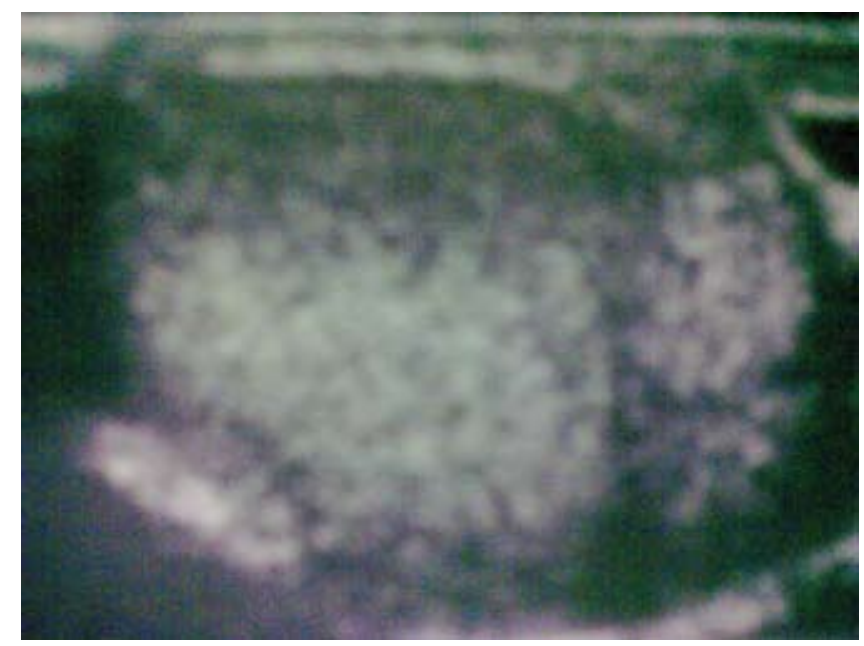

Figura 2 - Resonancia magnética en la que se evidencia el tumor adenomatoide.

B I B L I O G R A F Í A

1. Moyano Calvo JL, Giraldez Puig J, Sánchez de la Vega J, Dávalos Casanova G, Morales López A. Tumor adenomatoide de epidídimo. Actas Urol Esp. 2007;31(4):417-9.

2. Amin MB. Selected other problematic testicular and paratesticular lesions: rete testis neoplasms and pseudotumors, mesothelial lesions and secondary tumors Modern Pathol. 2005;18:S131-45.

3. Williams SB, Han M, Jones R, Andrawis R. Adenomatoid tumor of the testes. Urology. 2004;63(4):779-81.

4. Kontos S, Fokitis I, Karakosta A, Koritsiadis G, Mitsios K, Koutsikos S, et al. Adenomatoid tumor of epididymidis: A case report. Cases J. 2008;1:206.

5. Bestard Vallejo JE, Tremps Velázquez E, Blázquez Mañá C, Celma Doménech A, de Torres Ramírez I, Morote Robles J. Tumor adenomatoide de epidídimo: el tumor más frecuente de las estructuras paratesticulares. Actas Urol Esp. 2008;32(6):611-7.

6. Robert H. Young testicular tumors. Some new and a few perennial problems. Arch Pathol Lab Med. 2008;132:548-64.

7. Barry P, Chan KG, Hsu J, Quek ML. Adenomatoid tumor of the tunica albuginea. Int J Urol. 2005;12(5):516-8.

8. Patel MD, Silva AC. MRI of an adenomatoid tumor of the tunica albuginea. AJR Am J Roentgenol. 2004;182(2):415-7.

9. Flores N, Infante R. Tumores paratesticulares. En: Jiménez Cruz JF, Rioja Sanz LA, editores. Tratado de Urología. p. 2181-93.

10. Singh I, Dev G, Singh N. Chronic epididymitis (epididymal nodule) mimicking an adenomatoid tumor-case report with review of literature. Int Urol Nephrol. 2002;34(2):219-22.

P. Bahílo Mateu*, A. M. Soto Poveda, C. Di Capua Sacoto, G. García Fadrique, G. Morales Solchaga, J. Morera y J. F. Jiménez Cruz Servicio de Urología, Hospital Universitario La Fe, Valencia, España

${ }^{*}$ Autor para correspondencia. Correo electrónico: pilarbahilo@comv.es (P. Bahillo Mateu) 